\title{
Spinal cord transplants: a future treatment for spinal injury?
}

\author{
J Fawcett \\ Department of Physiology, University of Cambridge, Downing Street, Cambridge CB2 3EG, UK
}

The general principles of neural transplantation are now fairly well established, as a result of experience with transplanting many different regions of the central nervous system (CNS). A major stimulus to this work has been the finding that some of the symptoms of Parkinson's disease can be improved by grafts of embryonic substantia nigra, and many of the basic rules of transplantation have come out of the development of this procedure. In general only embryonic nervous tissue will survive transplantation, and this must be removed from the embryo during a narrow time window corresponding with the time at which the neurons in question have just undergone their final cell division. The transplanted neurons can grow processes into the host CNS, and make functional synaptic connections with host neurons. Similarly, host neuronal processes can sprout or regenerate into the transplant and make synapses with grafted neurons. The limitation, however, is that axons from the graft will not grow for appreciable distances into the host CNS unless there are target neurons immediately adjacent to the graft. In the case of substantia nigra grafts, for instance, if the graft is placed within the host substantia nigra, its axons will not grow through the host brain to their correct target in the striatum, they will only make local contacts. In order, therefore, to have an effect in Parkinson's disease, grafts must be placed heterotopically within the striatum, so that their axons can grow out and make connections. ${ }^{1-4}$ The extent of the connections made by grafts is very dependent on the age of the host. Grafts placed into newborn hosts, whose CNS has not yet become inhibitory to axon growth, grow axons extensively, and also receive a profuse axonal projection from the host neurons. However, as animals age, the glial environment of their CNS becomes inhibitory to axon growth, and the regenerative ability of their neurons decreases, and this is reflected in a diminution of connections to and from a graft of embryonic CNS tissue. ${ }^{5,6}$ Embryonic grafts, therefore, can in favourable circumstances replace the function of lost neurons. However, before they become useful for the treatment of most CNS disorders there are several practical problems which will need to be solved. These are (1) that a large proportion of grafted neurons may die soon after transplantation (around $97 \%$ in the case of substantia nigra transplants); (2) that axons from the grafts will not grow for long distances through non-target regions of the host CNS; and (3) that obtaining fresh embryonic tissue for each procedure places very severe logistical and ethical limitations on the procedure.

Neural transplantation has been under investigation for some years as a possible therapy for spinal cord injury, and there have recently been some encouraging results in animal models of the condition involving both transection and contusion injuries. ${ }^{5,7,8}$ Embryonic cord has been placed into cord lesions of various ages of host. In general, as with other types of CNS graft, those placed into newborn animals have made the most widespread and extensive connections with host cord neurons, the degree of connectivity falling with host age. However, even in adult hosts, there are still connections formed. Thus graft neurons have been shown to make local connections with host spinal cord neurons, and host neurons regenerate or sprout their axons into the grafts. ${ }^{8,9-12}$ Host axons show some differences in their growth potential in that sensory axons from dorsal root ganglion neurons grow extensively, while descending systems such as corticospinal fibres grow relatively sparsely. ${ }^{10}$ Different types of graft neuron also display different abilities to innervate the host; most graft-derived axons penetrate a maximum of $5 \mathrm{~mm}$ into host cord, but monoaminergic fibres may grow up to $15 \mathrm{~mm}$ in rats. ${ }^{5,13}$

The crucial question, however, is whether the grafts integrate functionally into the host CNS, and bring back lost functions. Physiological recordings have shown that stimulation of sensory fibres in the dorsal root can cause postsynaptic potentials in graft neurons, and anatomical studies show synapses made by hostand graft-derived axons, ${ }^{8}$ so functional connections are made, but are these connections behaviourally useful? There have recently been careful behavioural tests done on rats with partial cord lesions and embryonic grafts, which demonstrate that a variety of behaviours which are lost after cord section can show improvement after grafting. ${ }^{5}$ To what extent these behaviours rely on the reconstruction of local reflex circuitry and to what extent they depend on the resumption of control by descending axons from the brain is not entirely clear, but there is evidence that some of the forms of restored behaviour require descending control. These recent demonstrations of partial functional reconstruction of cord injury using graft technology lead one to expect that improvements in the technique will lead to better results in the future. 
Exactly how grafts might work is less clear. In principle there are several effects which might be expected. Grafts to the region of the injury could act as a bridge allowing axons to regenerate into the distal stump of the cord: this has been demonstrated when newborn rats are injured and grafted, but is not seen in adult animals. ${ }^{9,11}$ Grafted neurons could act as 'relays' by receiving connections from regenerating host axons, and passing on information by themselves making connections with host spinal cord neurons: this is the most likely mechanism for the recovery seen in grafts to adult animals. Grafts to the region of the injury could remyelinate the many demyelinated axons found passing through injured cord, or modify the environment around uncut but non-conducting axons so as allow them to regain their function: there is little evidence for or against these possibilities. The graft could be also acting as a source of neurotrophins, promoting survival of damaged host axons, and could be inhibiting the cavitation and glial scar formation which usually occurs: inhibition of scar and cavity formation certainly occurs, ${ }^{8,14}$ although its functional significance is unknown, and the role of secreted trophic factors is also not established.

In addition to embryonic neuronal grafts, there has been a considerable body of work on grafts specifically designed to encourage axon regeneration in the damaged cord. Almost all this work has focused on Schwann cells, since it is these cells which support axon regeneration in peripheral nerves and since implanted Schwann cells have been shown to promote regeneration of adult CNS axons. The most concerted effort in this direction has come from workers in the Miami Project to Cure Paralysis. They have been able to greatly expand Schwann cell numbers in tissue culture, then place them in a semi-permeable tube which is inserted into the damaged dorsal columns. Very large numbers of axons will regenerate into these grafts, but the problem remains that the regenerating axons show very little ability to leave the grafts and grow on into the spinal cord to make useful functional connections. ${ }^{15}$ This technology will therefore have to be combined with techniques to make the environment of the adult spinal cord less inhibitory to axon regeneration before function can be restored using these techniques. Embryonic spinal cord tissue may also act as a bridge to allow axons to regenerate across a lesion, but again these axons only progress beyond the bridge if the host is a newborn animal.

Spinal cord grafts have just started to reach the stage where they can reconstruct some of the circuitry in the damaged cord in experimental animals, with some behavioural benefit. The technology is barely ready for human patients, although some transplants have been performed in Russia, and limited improvement is reported over two to five spinal levels. ${ }^{7}$ However, from experience in Parkinson's disease one can predict that grafts are very unlikely to do any harm, and therefore experimental trials might be expected sooner rather than later. Will grafts ever be of benefit to the spinally injured patient? With the technology available now, and likely to become available in the next few years, the axonal connections made as a result of spinal cord grafting are likely to be restricted to the regions within a centimetre of the graft, and therefore effects will be local. However, there are many patients with high cervical lesions whose hand function might be greatly improved by regaining control of an extra spinal level or two. Effects on the rest of the cord might come from remyelination of the many locally demyelinated axons seen in most partial cord lesions, but the extent to which this can be achieved has yet to be established.

\section{References}

1 Brundin $\mathrm{P}$ et al. Survival and function of dissociated rat dopamine neurones grafted at different developmental stages or after being cultured in vitro. Dev Brain Res 1988; 39: 233-243.

2 Dunnett SB, Bjorklund A, Stenevi U. Dopamine-rich transplants in experimental Parkinsonism. TINS 1983; 6: 266-270.

3 Murphy $\mathbf{M}$ et al. FGF2 regulates proliferation of neural crest cells, with subsequent neuronal differentiation regulated by LIF or related factors. Development 1994; 120: 3519-3528.

4 Widner $\mathrm{H}$ et al. Bilateral fetal mesencephalic grafting in two patients with Parkinsonism induced by 1-methyl-4-phenyl1,2,3,6-tetrahydropyridine (MPTP). N Engl J Med 1992; 26: 1556-1563.

5 Bregman BS et al. Recovery of function after spinal cord injury: Mechanisms underlying transplant-mediated recovery of function differ after spinal cord injury in newborn and adult rats. Exp Neurol 1993; 123: 3-16.

6 Perry VH, Lund RD, McLoon SC. Ganglion cells in retinae transplanted to newborn rats. J Comp Neurol 1985; 231: 353-363.

7 Reier PJ et al. Workshop on intraspinal transplantation and clinical application. J Neurotrauma 1994; 11: 369-377.

8 Reier PJ, Stokes BT, Thompson FJ, Anderson DK. Fetal cell grafts into resection and contusion/compression injuries of the rat and cat spinal cord, Exp Neurol 1992; 115: 177-188.

9 Bernstein-Goral H, Bregman BS. Spinal cord transplants support the regeneration of axotomized neurons after spinal cord lesions at birth: A quantitative double-labeling study. Exp Neurol 1993; 123: 118-132.

10 Itoh Y, Tessler A. Regeneration of adult dorsal root axons into transplants of fetal spinal cord and brain-a comparison of growth and synapse formation in appropriate inappropriate targets, J Comp Neurol 1990; 302: 272-293.

11 Iwashita Y, Kawaguchi S, Murata M. Restoration of function by replacement of spinal cord segments in the rat. Nature 1994; 367: $167-170$.

12 Jakeman LB, Reier PJ. Axonal projections between fetal spinal cord transplants and the adult rat spinal cord: A neuroanatomical tracing study of local interactions. J Comp Neurol 1991; 307: 311-334.

13 Foster GA et al. Transmitter expression and morphological development of embryonic medullary and mesencephalic raphe neurones after transplantation to the adult rat central nervous system. Exp Brain Res 1985; 60: 427-444.

14 Krueger $\mathrm{S}$ et al. Three morphologically distinct types of interface develop between adult host and fetal brain transplants: implications for scar formation in the adult central nervous system. J Comp Neurol 1986; 249: 103-116.

$15 \mathrm{Xu}$ XM, Guénard V, Kleitman N, Bunge MB. Axonal regeneration into Schwann cell-seeded guidance channels grafted into transection adult rat spinal cord. J Comp Neurol 1995; 351: 145-160. 